"Mircea cel Batran" Naval Academy Scientific Bulletin, Volume XIX - 2016 - Issue 1

Published by "Mircea cel Batran" Naval Academy Press, Constanta, Romania // The journal is indexed in:

PROQUEST / DOAJ / DRJI / JOURNAL INDEX / I2OR / SCIENCE LIBRARY INDEX / Google Scholar / Crossref /

Academic Keys / ROAD Open Access / OAJI / Academic Resources / Scientific Indexing Services / SCIPIO

\title{
ABOUT THE STUDY OF ENERGY'S FLOW FROM ENERGETICALLY NAVAL SYSTEMS
}

\author{
Levent ALI ${ }^{1}$ \\ Anastase PRUIU ${ }^{2}$ \\ Beazit ALI ${ }^{3}$ \\ Daniel MĂRĂŞESCU ${ }^{4}$ \\ ${ }^{1} \mathrm{PhD}$ attendee Eng. Military Technical Academy \\ ${ }^{2}$ Professor PhD Eng., Marine Engineering and Naval Weapons Department \\ ${ }^{3}$ Professor PhD Eng., Marine Engineering and Naval Weapons Department \\ ${ }^{4} \mathrm{PhD}$ attendee Eng., Marine Engineering and Naval Weapons Department
}

Abstract: In this paper are analyzed energy's flow in energetically naval systems and their correlation with the effective power of thermal machines. The possibilities for secondary energy flows recovering and their impact on the marine environment with possibilities to reduce pollution

\section{INTRODUCERE}

In operating the plant or energy systems, a special attention must be given to mass flows and energy flows because they are main characteristic for a specific physical structure (sections flow of piping, sections of flow through thermal machines and working, sections of energetically exchanges, the components of the energy fluids, etc.).

\section{MASS FLOWS}

$\dot{m}_{C H}\left[\frac{K g_{c b}}{b}\right]$ - fuel consumption

$\tilde{m}_{A H}\left[\frac{R_{g a r r}}{n}\right]$ - air flow

$\dot{m}_{\text {AHSG }}\left[\frac{m_{\text {axr }}}{m_{\text {an }}}\right]$ - air flow for gas exchange

$\dot{m}_{\text {AHARD }}\left[\frac{k g_{\text {axr }}}{n}\right]$ - air flow for combustion

$\tilde{m}_{G H}\left[\frac{\mathrm{Kg}_{\operatorname{garr}}}{\mathbb{R}}\right]$ - gas exhaust flow

$$
\dot{m}_{C H}=c_{e} \cdot P_{\theta}\left[\frac{K g_{c b}}{i n}\right]
$$

$c_{\theta}\left[\frac{K g_{c b}}{k W h}\right]-$ effective specific fuel consumption

$\mathrm{Pe}[\mathrm{kW}]$ - effective power

$$
\tilde{m}_{A H}=\tilde{m}_{C H} \times \alpha \cdot m_{\text {aErt }}\left[\frac{K g_{a x r}}{\hbar k}\right]
$$

$\alpha$ - excess air coefficient

$\alpha_{\mathrm{SG}}$ - excess air coefficient for gas exchange

$\alpha_{\text {ARD }}$ - excess air coefficient for combustion

$$
\begin{aligned}
& \dot{m}_{\text {AHSG }}=\dot{m}_{C H} \times \alpha_{S G} \times m_{\text {aErt }}\left[\frac{K g_{a g r}}{k}\right][3]
\end{aligned}
$$

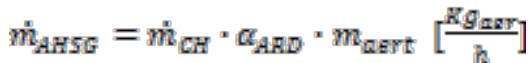

$m_{\text {aert }}\left[\mathrm{Kg}_{\mathrm{aer}} / \mathrm{Kg}_{\mathrm{cb}}\right]$ - minimum theoretical air mass for a full combustion a kilogram of fuel

$$
\begin{aligned}
& \dot{m}_{G H}=\dot{m}_{C H}+m_{\text {aert }}\left[\frac{K_{g \text { garg }}}{\hbar}\right] \\
& \dot{m}_{G H}=\dot{m}_{C H} \cdot\left[1+\alpha_{S G} \cdot m_{\text {aErt }}\right]\left[\frac{K g_{g a r g}}{\hbar}\right]
\end{aligned}
$$

Specific units

$c_{\varepsilon}\left[\frac{K g_{c b}}{k W h}\right]-$ effective specific fuel consumption

$d_{a s g}\left[\frac{K g_{a v r}}{k W h}\right]$ - specific air flow for gas exchange

$$
d_{\text {agg }}=c_{\theta} \times \alpha_{S G} \times m_{\text {aert }}\left[\frac{\mathrm{Kg}_{\mathrm{agr}}}{\mathrm{n}}\right]
$$

$$
\begin{aligned}
& d_{\text {aard }}\left[\frac{K g_{a v r}}{k W h}\right]-\text { specific air flow for combustion } \\
& d_{\text {aard }}=c_{\varepsilon} \times \alpha_{A R D} \cdot m_{\text {aert }}\left[\frac{\mathrm{K} g_{\text {arr }}}{\mathbb{R}}\right] \\
& \mathrm{D}_{\mathrm{g}}[\mathrm{Kg} \text { gaze } / \mathrm{kWh}] \text { - specific gas flow } \\
& d_{g}=c_{\theta}+d_{\text {asg }}\left[\frac{K g_{g a r x v}}{\pi}\right] \\
& d_{g}=c_{\theta} \cdot\left[1+\alpha_{S G} \cdot m_{\text {aert }}\right]\left[\frac{K g_{g a r g}}{\hbar k}\right]
\end{aligned}
$$

\section{ENERGY FLOWS}

\subsection{Energy flow available}

$$
\phi_{d}^{i}=\frac{m_{\mathrm{cH}} Q_{\mathrm{i}}}{3600}[\mathrm{~kW}]
$$

$\mathrm{Q}_{i}\left[\mathrm{~kJ} / \mathrm{Kg}_{\mathrm{cb}}\right]$ - lower calorific power

\subsection{Effective power}

$$
\begin{aligned}
& P_{\theta}=\eta_{e} \cdot Q_{d}[\mathrm{~kW}] \\
& \eta_{e}=\frac{P_{Q}}{Q_{d}}=\eta_{\mathrm{i}} \cdot \eta_{e}
\end{aligned}
$$

$\eta_{\mathrm{e}}$ - effective efficiency

$\eta_{i}$ - medium indicated efficiency (each cylinder have an indicated efficiency)

$$
\eta_{m}=\frac{p_{g}}{p_{i}}=\frac{c_{i}}{c_{g}}=\frac{L_{\underline{g}}}{L_{i}}
$$

$\eta_{m}-$ mechanical efficiency

$\mathrm{P}_{\mathrm{i}}[\mathrm{kW}]$ - indicated power

$$
P_{\mathrm{i}}=\sum P_{\text {icil }}[\mathrm{kW}]
$$

$P_{\text {icil }}[k W]$ - indicated power for each cylinder

$c_{j}\left[\frac{K g_{c b}}{k W h}\right]$ - indicated specific fuel consumption

$\mathrm{L}_{\mathrm{e}}[\mathrm{kNm}]$ - effectively machine work

$\mathrm{L}_{i}[\mathrm{kNm}]$ - indicated machine work

Observations: if the effective operating power is low, or the engine is operating at no-load running must be used indicated specific fuel consumption.

\subsection{Operating Effective Power}

$P$ en $[k W]$ - nominal effective power (the engine have the nominal rpm, in environmental standard condition with fuel $Q_{i}=42700\left[\mathrm{~kJ} / \mathrm{Kg}_{\mathrm{cb}}\right]$ )

$P_{\text {eexp }}[k W]$ - operating effective power

$P_{\text {exp }}=0[\mathrm{~kW}]-$ no-load for engine

$$
P_{\text {eexpmax }}=1.1 \times P_{\text {en }}[\mathrm{kW}]
$$

$P_{\text {eexpmax }}$ - maximum operating effective power 
"Mircea cel Batran" Naval Academy Scientific Bulletin, Volume XIX - 2016 - Issue 1

Published by "Mircea cel Batran" Naval Academy Press, Constanta, Romania // The journal is indexed in:

PROQUEST / DOAJ / DRJI / JOURNAL INDEX / I2OR / SCIENCE LIBRARY INDEX / Google Scholar / Crossref /

Academic Keys / ROAD Open Access / OAJI / Academic Resources / Scientific Indexing Services / SCIPIO

$$
\mathrm{K}_{\mathrm{p}}=\mathrm{P}_{\mathrm{eexp}} / \mathrm{P}_{\mathrm{en}}
$$

$\mathrm{K}_{\mathrm{p}}$ - loading factor or power ratio;

$\mathrm{K}_{\mathrm{p}}=0$ - for no-load;

$\mathrm{K}_{\mathrm{p}}=1-$ for nominal load;

$\mathrm{K}_{\mathrm{p}}=1.1-$ for maximum load

According with [B4] for ship propulsion the

following engine types are used:

- Slow speed two stroke diesel engines (50 - 300

RPM)

- Medium speed four stroke diesel engines (300 1000 RPM)

- Gas turbines (very high RPM > 5000)

Approximate/typical SFOC values for different engine types (at $42.7 \mathrm{MJ} / \mathrm{kg}$ oil)

Slow speed engines: $\quad 155-175[\mathrm{~g} / \mathrm{kWh}]$

Medium speed engines: $175-200[\mathrm{~g} / \mathrm{kWh}]$

High speed engines: $\quad 195-225[\mathrm{~g} / \mathrm{kWh}]$

Gas turbines:

$240-300[\mathrm{~g} / \mathrm{kWh}]$

\subsection{Energy flows for turbocharger}

Available energetic flow discharged from cylinders with exhaust gas.

$$
\phi_{g}=\frac{m_{C H^{\prime}} C_{F C}\left(t_{g a v}-t_{0 r a f}\right)}{a 600}[k W]
$$

$\mathrm{C}_{P G}\left[\mathrm{~kJ} / \mathrm{Kg}_{G} \cdot{ }^{\circ} \mathrm{C}\right]-$ specific heat of exhaust gas

$$
c_{P G}=\sum c_{P G i} \cdot g_{\mathrm{i}}\left[\frac{\mathrm{kJ}}{\mathrm{Kg}^{* A C} \mathrm{C}}\right]
$$

$\mathrm{C}_{\mathrm{PGj}}\left[\mathrm{kJ} / \mathrm{Kg}_{G} \cdot{ }^{\circ} \mathrm{C}\right]$ - specific heat for each component of exhaust gas

$g_{j}=\frac{m_{g j}}{m_{g}}-$ mass composition of each component of exhaust gas

$\dot{m}_{g j}$ - flow for each component of exhaust gas

$t_{\text {gev }}\left[{ }^{\circ} \mathrm{C}\right]$ - medium temperature for outlet the cylinder of exhaust gas

$t_{\text {oref }}\left[{ }^{\circ} \mathrm{C}\right]$ - standard or reference environmental air temperature

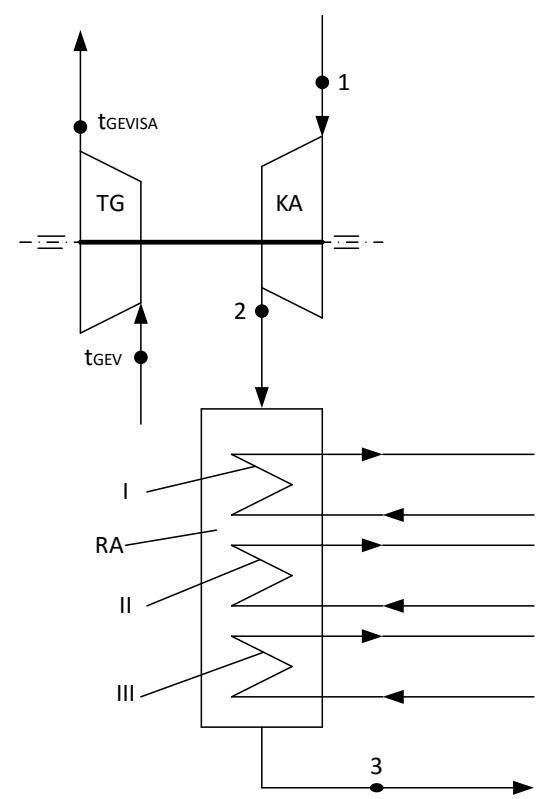

TG - gas turbine from turbocharger
$\mathrm{KA}$ - air compressor from turbocharger

RA - air cooler

1 - air compressor suction

2 - air compressor discharge

3 - outlet air from scavenge air cooler

I - cooler with fresh water (preheat system stage from auxiliary boiler)

II - cooler with fresh water (stage from fresh water system)

III - cooler with sea water - oversize cooler stage adjustment

The ambient reference conditions according to IACS URM28 are the regular machinery space room temperature $45^{\circ} \mathrm{C}$, ambient pressure 1,000 mbar absolute and air humidity $60 \%$.

\begin{tabular}{|l|l|l|l|}
\cline { 2 - 4 } \multicolumn{1}{c|}{} & 1 & 2 & 3 \\
\hline $\mathrm{t}\left[{ }^{\circ} \mathrm{C}\right]$ & 45 & 150 & 60 \\
\hline $\mathrm{T}[\mathrm{K}]$ & 318 & 423 & 333 \\
\hline $\mathrm{p}[\mathrm{bar}]$ & 1 & 3 & 3 \\
\hline $\mathrm{p}\left[\mathrm{kN} / \mathrm{m}^{2}\right]$ & 100 & 300 & 300 \\
\hline$\rho\left[\mathrm{Kg}\right.$ aer $\left./ \mathrm{m}^{3}\right]$ & 1.099 & 2.48 & 3.49 \\
\hline
\end{tabular}

$T_{2}=T_{1} \cdot\left(\frac{p_{2}}{p_{1}}\right)^{\frac{m_{g}-1}{m_{s}}}[K] ; n_{g}-$ politropic coefficient; $\mathrm{p}_{2}$ - required for turbocharger;

Ex: $\quad \mathrm{p}_{2}=3$ bar; $n_{g}=1.35$; $T_{2}=318 \cdot\left(\frac{a}{1}\right)^{\frac{1.35-1}{1.35}}=423[\mathrm{~K}] ; \mathrm{T}_{3}-$ depended with temperature suction, discharge pressure and relative humidity $\varphi[\%]$

$T_{a}=T_{a}+[3 \div 20][K]$ - to avoid a massive condensation of water vapor from air

$\rho=\frac{p}{R T} ; \mathrm{R}=0.286[\mathrm{~kJ} / \mathrm{KgK}]$

turbine

Energetic flow discharged from gas

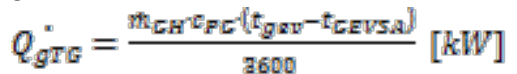

$\Delta t_{\text {GTG }}=t_{\text {GEV }}-t_{\text {GEVSA }}\left[{ }^{\circ} \mathrm{C}\right]-$ temperature decrees in gas turbine

Energetic flow of air receives in air compressor

$$
Q_{A K A}^{\circ}=\frac{m_{A H S E} c_{P A}\left(t_{r a f}-t_{0}\right)}{3600}[k W]
$$

Effective power of gas turbine is equal by compressor driven power

Results:

$$
P_{\text {eTG }}=P_{\text {antKA }}[\mathrm{kW}]
$$

$$
Q_{g T G}^{*} \eta_{T G}=\frac{Q_{A K A}^{*}}{\eta_{K A}}[k W]
$$

the resulting:

or

$$
\Delta t_{\text {GTG }}=\frac{1}{\prod_{T C} \prod_{K A}} \cdot \frac{m_{A H S G}}{m_{C H}} \cdot \frac{c_{P A}}{c_{F C}} \cdot \Delta t_{A K A}\left[{ }^{\circ} \mathrm{C}\right]
$$

$$
\Delta t_{G T G}=\frac{1}{\eta_{T C C} \eta_{\mathrm{W} A}} \cdot \frac{d_{A S E}}{d_{C}} \times \frac{c_{P A}}{c_{F C}} \cdot \Delta t_{A K A}\left[{ }^{\circ} C\right]
$$

$$
\text { Numerical application }
$$

$\eta_{T G}=0.75 \div 0.85-$ gas turbine efficiency

$\eta_{\text {KA }}=0.75 \div 0.85-$ air compressor efficiency 
"Mircea cel Batran" Naval Academy Scientific Bulletin, Volume XIX - 2016 - Issue 1

Published by "Mircea cel Batran" Naval Academy Press, Constanta, Romania /I The journal is indexed in:

PROQUEST / DOAJ / DRJI / JOURNAL INDEX / I2OR / SCIENCE LIBRARY INDEX / Google Scholar / Crossref /

Academic Keys / ROAD Open Access / OAJI / Academic Resources / Scientific Indexing Services / SCIPIO

$d_{\text {ASG }}=0.18 \cdot 3 \cdot 13.5=7.29\left[\frac{\mathrm{Kg} \operatorname{sar}}{\mathrm{KWh}}\right]$;

$d_{G}=7.47\left[\frac{\mathrm{Kg} g a r s}{\mathrm{kWh}}\right] ; c_{P A}=1\left[\frac{\mathrm{kJ}}{\mathrm{Kg} g^{\circ C}}\right] ; c_{P G}=1.2\left[\frac{\mathrm{kJ}}{\mathrm{Kg} / \mathrm{C}}\right]$;

$\Delta t_{\text {GTG }}=\frac{1}{0.80 .85} \times \frac{7.29}{7.47} \times \frac{1}{1.2} \times 10^{5}=126\left[{ }^{\circ} \mathrm{C}\right] \quad-\quad$ this

value it is verified with naval diesel engine

Observations: the operating cases in which recovery boiler does not work, the temperature control stage with seawater, must be discharge an energy flow to allows a temperature decrees like $\Delta t_{A R} \cong 90{ }^{\circ} \mathrm{C}$.

The energy flow that will be transferred to seawater

$$
Q_{A R}^{\infty}=\frac{m_{A H S C} C_{P A} \Delta t_{A R}}{3600}[k W]
$$

\section{MASS FLOW OF CARBO DIOXIDE}

By the elemental analysis of fuel, known carbon content:

$c=0.85 \div 0.87\left[\frac{\mathrm{KgC}}{\mathrm{Kgcb}}\right]-$ for liquid fuel

Combustion:

$$
\mathrm{C}+\mathrm{O}_{2}+\mathrm{CO}_{2}+Q_{\mathrm{c}}
$$

$Q_{c}$ - the energy obtained by combustion

$$
\left\{\begin{array}{c}
1 \mathrm{Kmol} \mathrm{C}+1 \mathrm{Kmol} \mathrm{O}_{2} \rightarrow 1 \mathrm{Kmol} \mathrm{CO}_{2} \\
12 \mathrm{KgC}+32 \mathrm{Kg} \mathrm{O}_{2}=44 \mathrm{Kg} \mathrm{CO}_{2} \\
c \mathrm{KgC}+\frac{\mathrm{a2}}{12} c \mathrm{Kg} \mathrm{O}_{2}=\frac{44}{12} c \mathrm{Kg} \mathrm{CO}_{2}
\end{array}\right.
$$

Results:

$$
\dot{m}_{\mathrm{CO2}}=\frac{44}{12} \cdot c \cdot \dot{m}_{\mathrm{CH}}\left[\frac{\mathrm{Kg}}{\hbar}\right]
$$

specific mass of carbo dioxide

or

$$
d_{\mathrm{CO2}}=\frac{44}{12} \cdot c \cdot c_{\theta}\left[\frac{\mathrm{Kg} / \mathrm{CO2}}{\mathbb{R} W h}\right]
$$

specific flow of carbo dioxide

Numerical application

$d_{\text {CO2 }}=\frac{44}{12} \times 0.86 \cdot 0.18=0.57\left[\frac{K_{g_{\mathrm{CO} 2}}}{\mathrm{kWh}}\right]$

For a $35000[\mathrm{dwt}]$ vessel; $\mathrm{W}_{\mathrm{n}}=15[\mathrm{Nd}]$

$P_{e}=10000[\mathrm{~kW}]$

$\dot{m}_{\mathrm{CO2}}=d_{\mathrm{CO2} 2} \cdot P_{e}=0.57 \cdot 10000=5700\left[\frac{K_{g_{\mathrm{CO} 2}}}{h}\right]$

$$
\begin{aligned}
& d_{C O 2}=\frac{m_{\mathrm{CO2}}}{T_{\mathrm{m}} \times W_{\mathrm{n}}}=\frac{5700}{35000 \cdot 15}=0.01086\left[\frac{K_{g_{\mathrm{CO} 2}}}{t \cdot M n}\right] \\
& =10.86\left[\frac{g_{\mathrm{CO2} 2}}{t \cdot \mathrm{Mn}}\right]=5.86\left[\frac{g_{\mathrm{CO2}}}{t \cdot \mathrm{Km}}\right]
\end{aligned}
$$

[B1]Based on a HFO fuel saving of 3,555 tons per year (with $3 \%$ sulphur content), the installation of a WHRS on a large container ship, will save the environment for the following emission amounts:

CO2 emission saving per year: 11,260 tons

NOx emission saving per year: 319 tons

SOx emission saving per year: 214 tons

Particulates saving per year: 29 tons

[B2]The objective of regulations introduced by the International Maritime Organization (IMO), the European Union (EU), the US Environmental Protection Agency (EPA) and the California Air Resources Board (CARB) is to reduce the contribution shipping makes to global and local emissions.

Ship designers, owners and operators have three general routes to achieve SOx regulatory compliance:

- Use low sulfur residual or distillate marine fuels in existing machinery,

- Install new machinery (or convert existing machinery where possible) designed to operate on an inherently low sulfur alternative fuel, such as liquefyor natural gas (LNG),

- Install an exhaust gas cleaning (EGC) after treatment system.

Fuel Oils Sulfur Limits

\begin{tabular}{|l|l|l|}
\hline & GLOBAL & ECA \\
\hline Initial limits & $4.5 \%$ & $1.5 \%$ \\
\hline 1 Julay 2010 & $4.5 \%$ & $1,0 \%$ \\
\hline 1 Julay 2012 & $3.5 \%$ & $1.0 \%$ \\
\hline 1 Julay 2015 & $3.5 \%$ & $0.1 \%$ \\
\hline 1 Julay 2020 & $0.5 \%$ & $0.1 \%$ \\
\hline
\end{tabular}

\section{CONCLUSIONS}

a. Determining the mass flows by choosing the flow rate of fluid, allow thepiping designed and circulation sections of thermal machines;

b. On the main flow sections, respectively on the energy flows exchangers must be installed thermometers order to allow proper adjustment of the energy system and performing balance energy;

c. Allows the high rate pollution of ambient environmental components and the rate of ageing for different parts of the thermal machine are determinate by the composition of exhaust gases from thermal machines d. Operation of energy systems must respect fully statutory provisions concerning for reduction of pollution of the marine environment for the respective navigation areas;

e. Adjusting the energy flows take into account the navigation conditions being imposed by safety crew, vessel and cargo.

\section{BIBLIOGRAPHY}

[1] Waste Heat Recovery System (WHRS) for Reduction of Fuel Consumption, Emission and EEDI

[2] ABS - Exhaust Gas Scrubber Systems Advisory

[3]Exhaust Gas Emission Control Today and Tomorrow 
"Mircea cel Batran" Naval Academy Scientific Bulletin, Volume XIX - 2016 - Issue 1 Published by "Mircea cel Batran" Naval Academy Press, Constanta, Romania // The journal is indexed in: PROQUEST / DOAJ I DRJI / JOURNAL INDEX I I2OR / SCIENCE LIBRARY INDEX / Google Scholar / Crossref I Academic Keys I ROAD Open Access / OAJI / Academic Resources / Scientific Indexing Services / SCIPIO

[4] ENERGY DEMAND AND EXHAUST GAS EMISSIONS OF MARINE ENGINES, Technical University of Danemark Hans Otto Kristensen

[5] Study of Exhaust Gas Cleaning Systems for vessels to fulfill IMO III in 2016

[6] Safety Concept - Marine, Dual-fuel engines, Safety Concept, EN 